\title{
Induction and Tunability of Self-Healing Property of Dendron based Hydrogel using Clay Nanocomposite
}

\author{
Balachandran Vivek, Prashant kumar and Edamana Prasad* \\ Department of Chemistry, Indian Institute of Technology \\ Madras, Chennai 600 036, India \\ *E-mail: pre@,iitm.ac.in
}




\section{Supporting Information}

$\begin{array}{ll}\text { 1. Preparation procedure of Na-MMT } & \text { S3 }\end{array}$

2. Preparation of Swelled Na-MMT $\quad$ S3

3. Preparationof polycyclic aromatic hydrocarbon solutions $\quad$ S3

$\begin{array}{ll}\text { 4. Adsorption procedure } & \text { S3 }\end{array}$

5. Preparation of poly(aryl ether) gel $\quad$ S4

6. FT-IR studies of poly(aryl ether) gel $\quad$ S5

7. Thermal analysis of QPD-MMT (generation 1) S6

8. SEM images of nanocomposites and nanocomposite gel $\quad$ S7

9. TEM images of nanocomposites and nanocomposite gel $\quad$ S8

10. Time sweep curve of QPD-MMT-PAD $\quad$ S9

$\begin{array}{ll}\text { 11. Self-Healing studies } & \text { S10 }\end{array}$

12. Adsorption studies. $\quad$ S11 
1. Preparation of Na-MMT. MMT (1g) clay was dispersed in $100 \mathrm{ml}$ doubly distilled water, $\mathrm{NaCl}(1 \mathrm{~g})$ was added in the above solution. The above mixture was stirred at 80 ${ }^{\circ} \mathrm{C}$ for 3 days, filtered and washed with hot water for seven times to remove chloride ions and dried in vacuum . The same procedure was repeated 3 times, the clay after the whole procedure was used for organic intercalation.

2. Swelling of Na-MMT. Na-MMT (1g ) was stirred in doubly distilled water for one week and filtered using vacuum pump and dried in vacuum.

3. Preparation of Polycyclic aromatic hydrocarbon (PAH) solutions- polycyclic aromatic hydrocarbons (pyrene, $\beta$-naphthol and phenanthrene) in ppm level was obtained by dissolving $10 \mathrm{mg}$ the hydrocarbons in $1000 \mathrm{ml}$ doubly distilled water and diluted to1ppm for studies.

4. Adsorption of PAH - The solution containing pyrene, $\beta$-naphthol and phenanthrene (1ppm) was stirred with dried gel for $1 \mathrm{~h}$.The concentration of PAH were determined by fluorescence spectra. 
5. Preparation of poly(aryl ether) gel

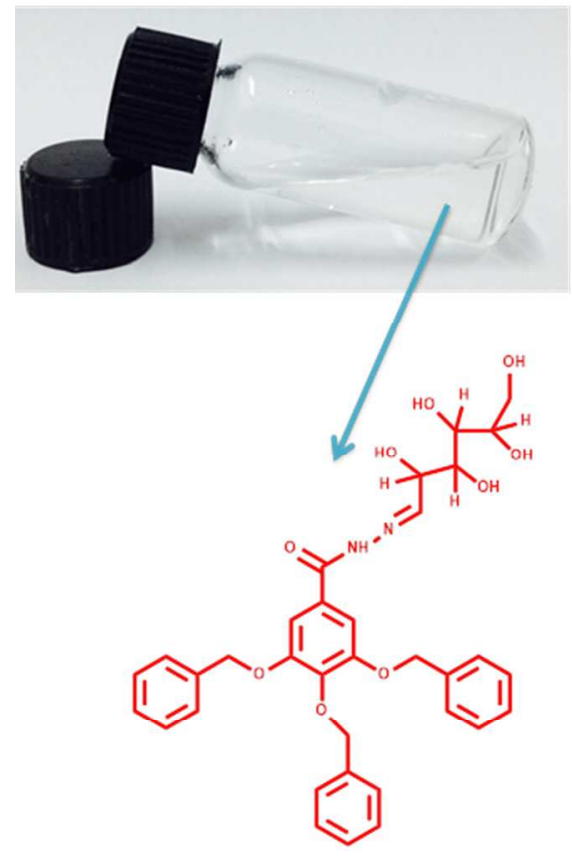

DMSO/Water (1:1)

Sonication

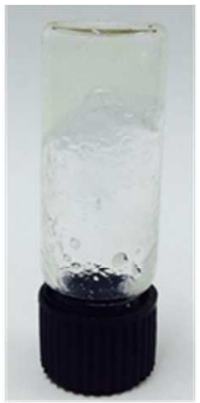

Scheme S1: Preparation of poly (aryl ether dendron) based low molecular weight gels. 
6. FT-IR studies of poly (aryl ether) gel

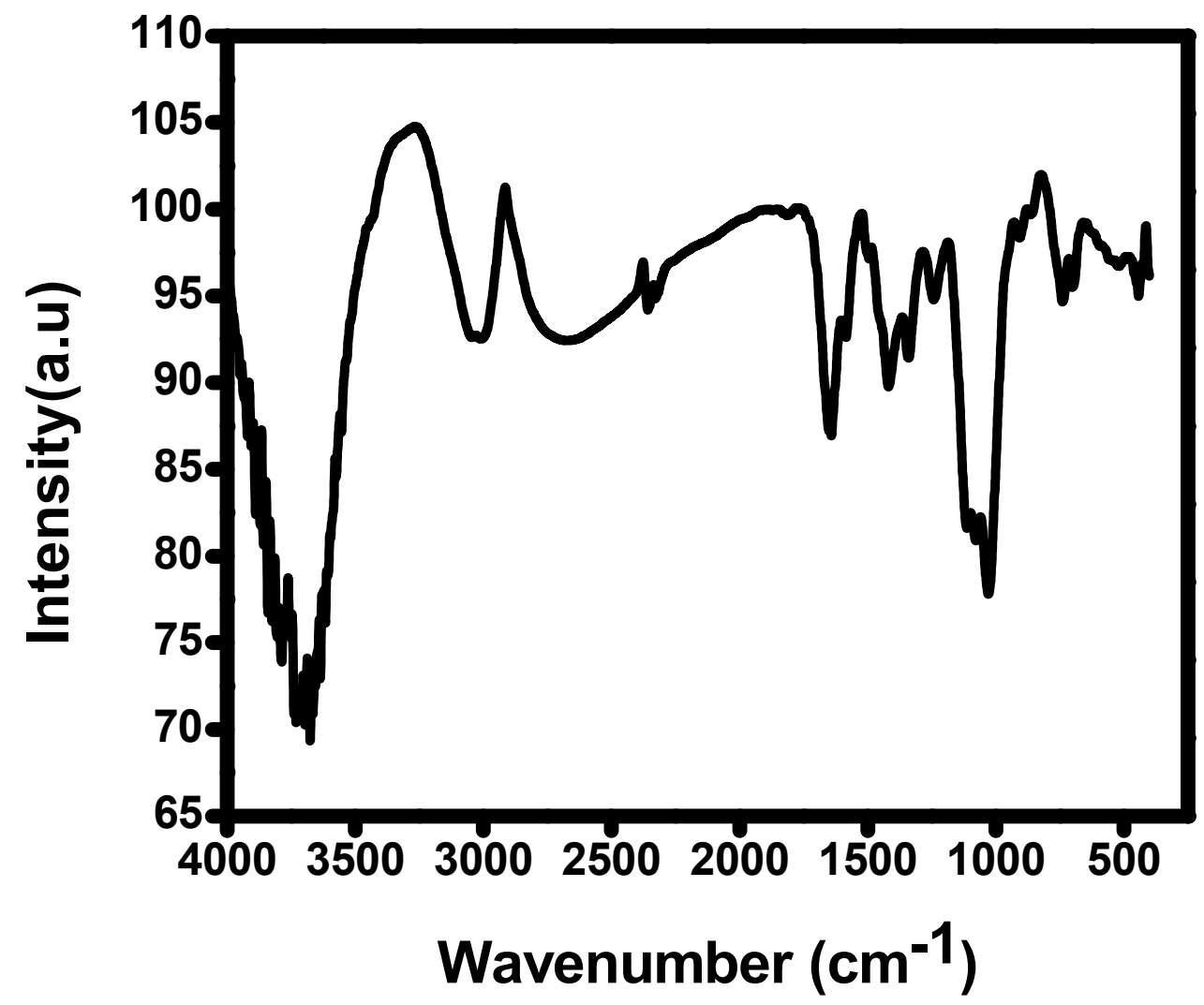

Figure S1: FT-IR spectrum of poly (aryl ether) gel. 
7. Thermal analysis of QPD-MMT (generation 1)
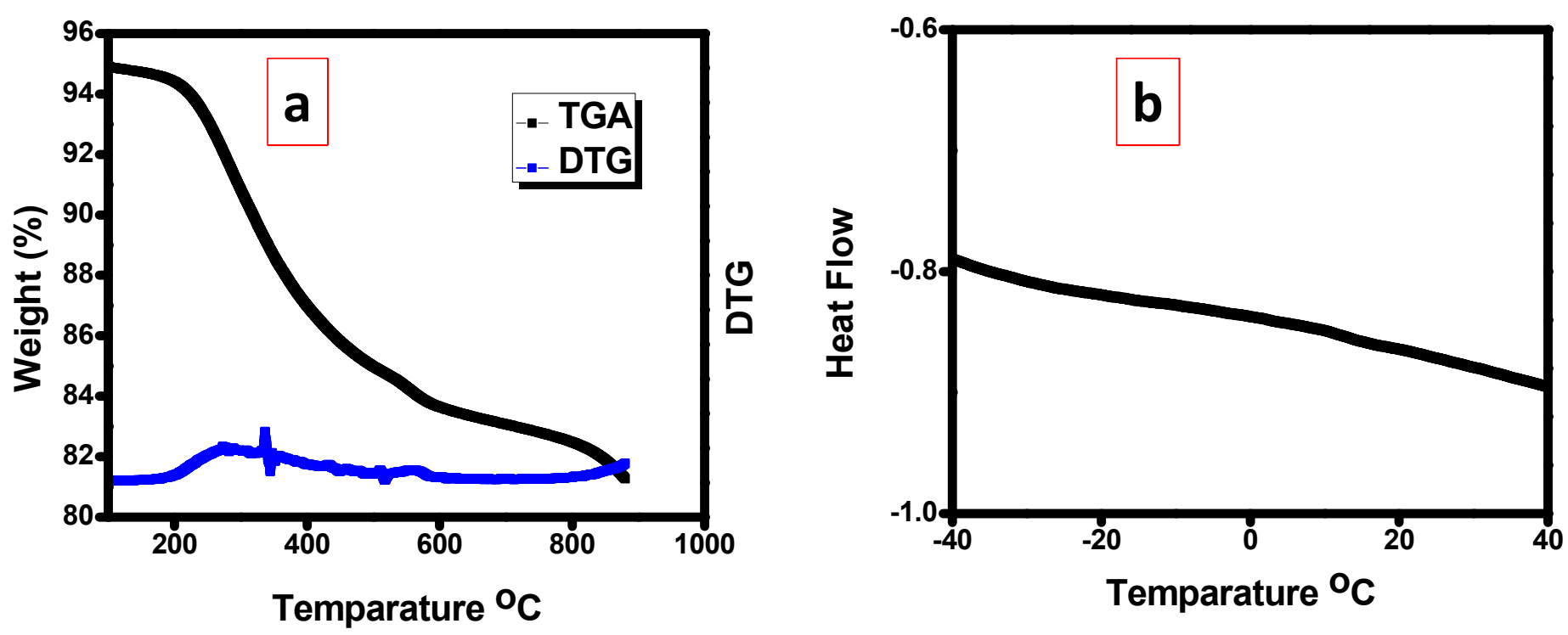

Figure S2: (a) TGA and (b) DSC of QPD-MMT (generation 1). 
8. SEM images of nanocomposites and nanocomposite gel
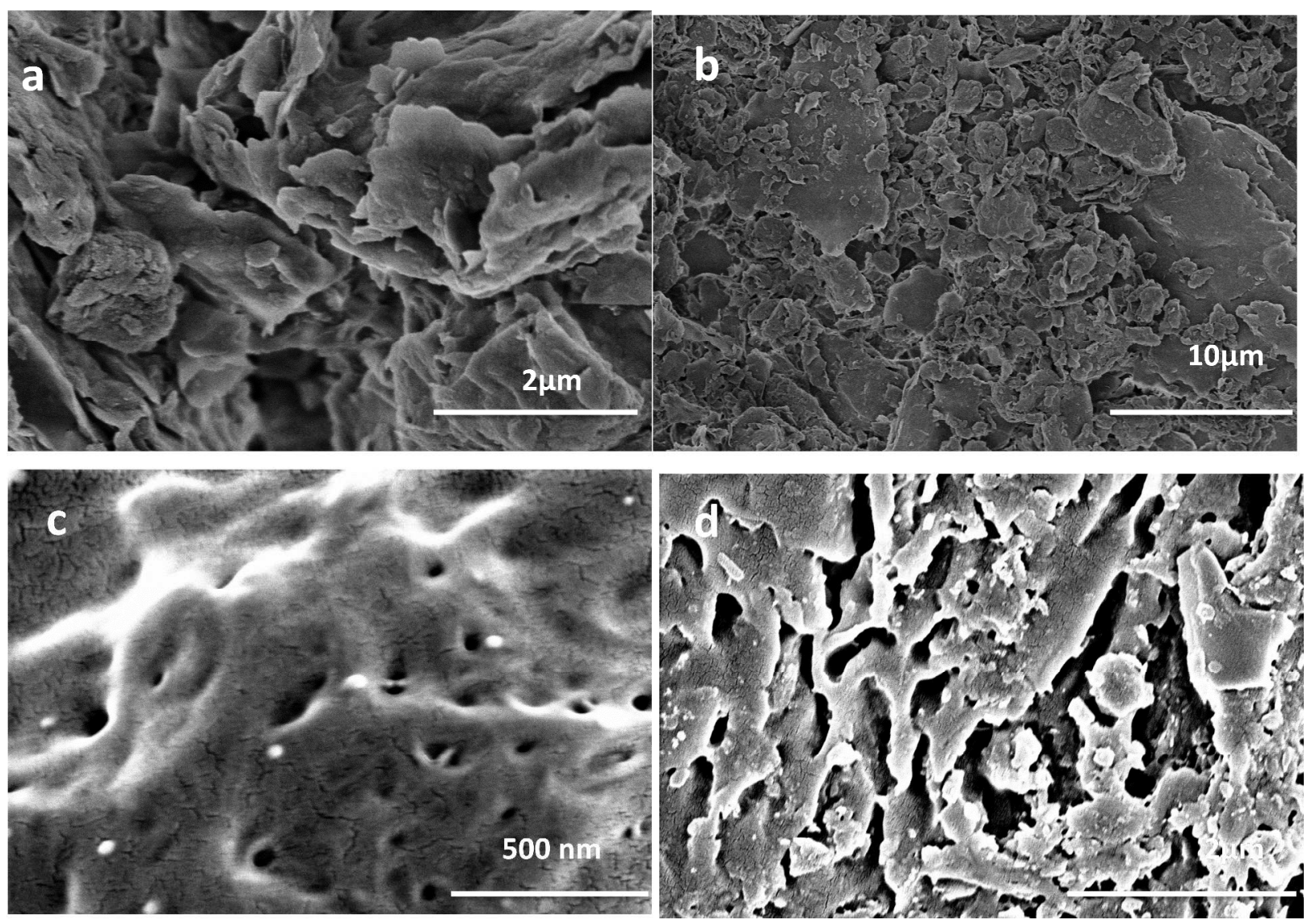

Figure S3: SEM images of (a) MMT (b) QPD-MMT(c) PAD (d) QPD-MMT-PAD. 


\section{TEM images of nanocomposites and nanocomposite gel.}

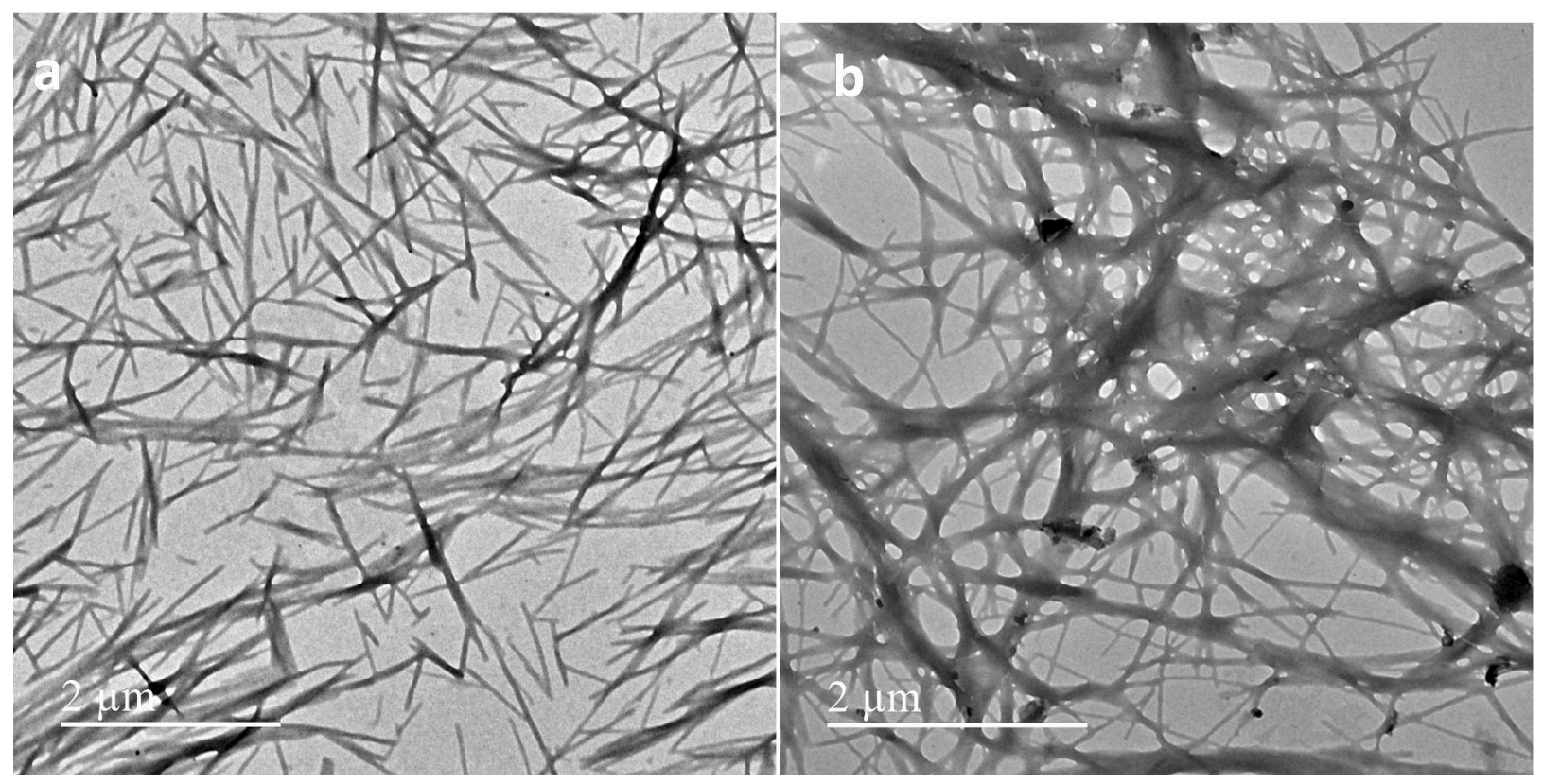

Figure S4: TEM images of (a) PAD and (b) QPD-MMT-PAD. 
10. Time sweep curve of QPD-MMT-PAD

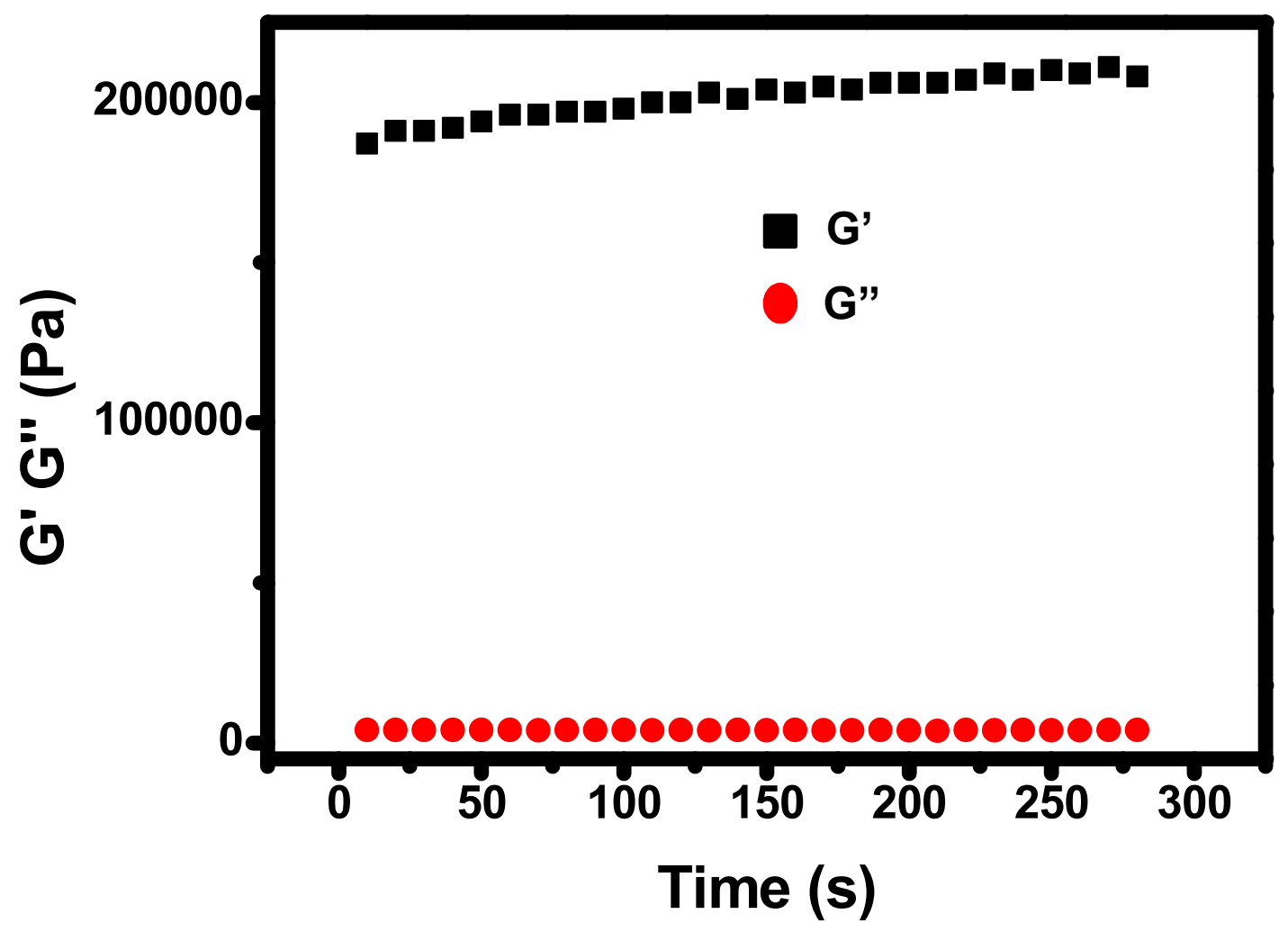

Figure S5: Time sweep curve of (G, G'” versus time) QPD-MMT-PAD. 
11. Self-Healing studies

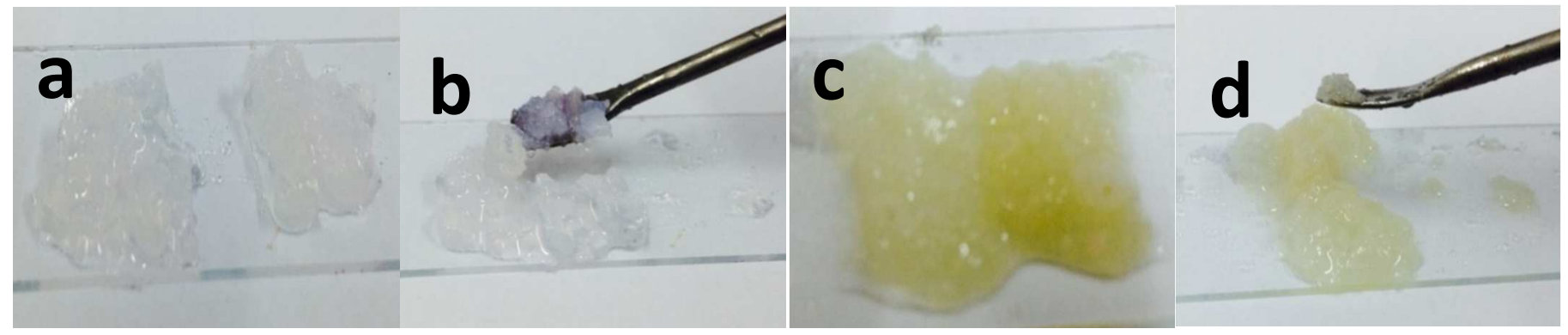

Figure S6: (A and b) Poor self-healing ability of PAD and (c and d) PAD with MMT. 


\section{Adsorption studies}
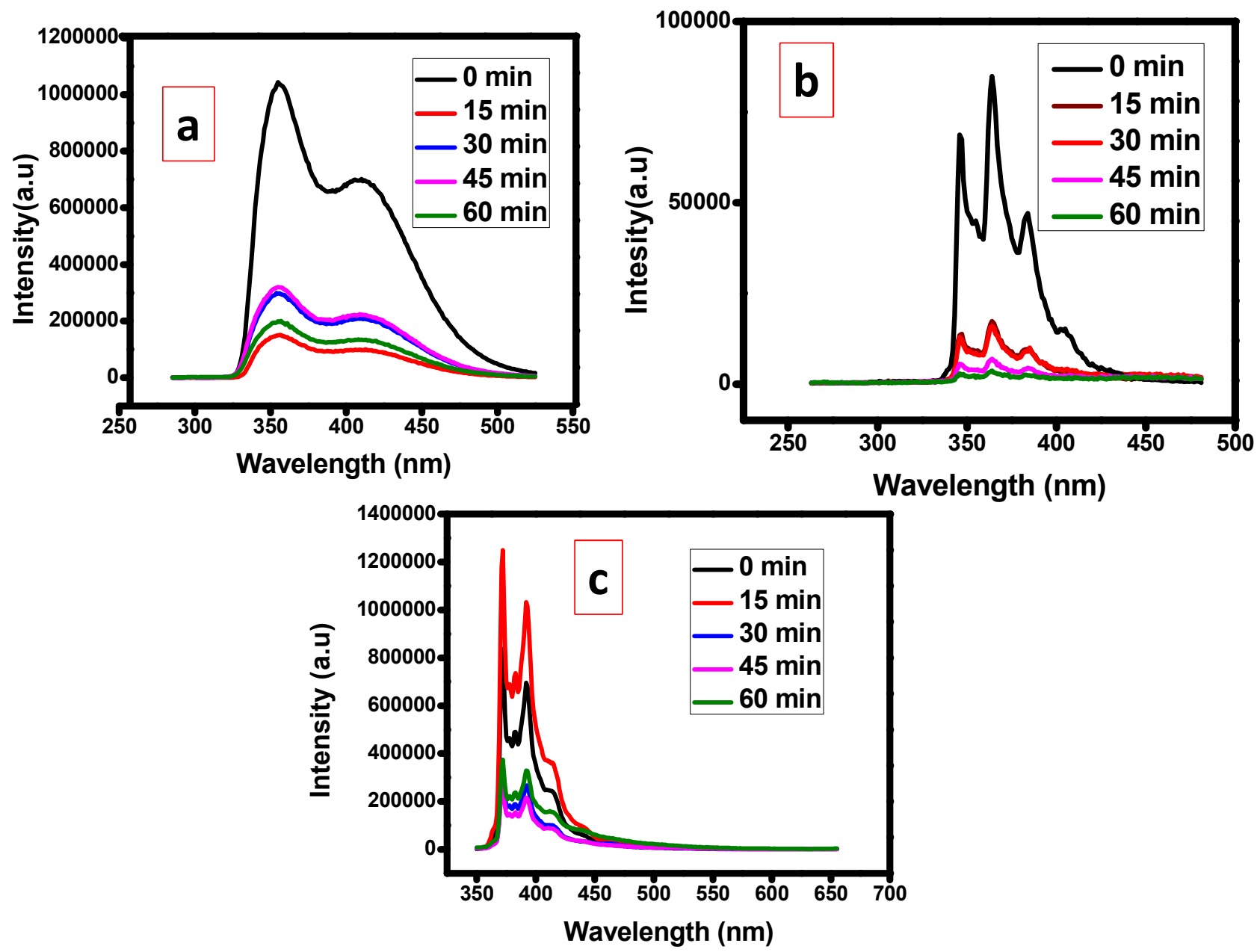

Figure S7: Fluorescence spectra of PAH \{(a) $\beta$-naphthol, (b) phenanthrene, (c) pyrene $\}$ during adsorption experiment. 


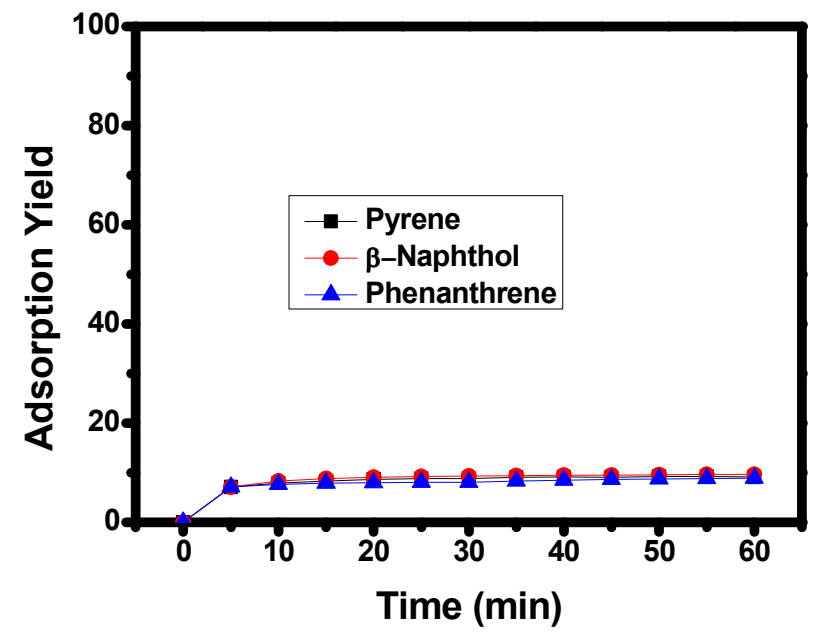

Figure S8: Adsorption versus time plot of PAH pyrene, $\beta$-naphthol and phenanthrene using nanoclays. 